\title{
LA FEMINIDAD NORMATIVA Y LA VIOLENCIA SEXUAL EN EL III REICH. LA DECONSTRUCCIÓN DE LAS IDENTIDADES FEMENINAS Y LA EXPLOTACIÓN SEXUAL DE LAS MUJERES EN LOS CAMPOS DE CONCENTRACIÓN Y EXTERMINIO
}

\author{
Femininity normative and sexual violence in the III Reich. \\ The deconstruction of femenine identity and the sexual exploitation of Jewish women in \\ the concentration and extermination camps
}

Yolanda BeteTA MARTín Universidad Complutense de Madrid (UCM) yolandabeteta@hotmail.com

Fecha de recepción: 15-II-2012 Fecha de aceptación: 15-IV-2012

RESUMEN: El artículo ofrece una visión general de la violencia ejercida sobre las mujeres en los campos de concentración y exterminio nazis a través de los testimonios ofrecidos por las propias supervivientes. Las mujeres, por el simple de serlo, padecen un tipo de violencia específica que tiene una doble significación: política y simbólica. Por ello la violencia sexual deba ser analizada como una categoría de análisis propia que incorpore una perspectiva de género. El artículo se estructura en dos partes diferenciadas e interdependientes. En primer lugar y a modo de introducción se analiza el discurso nacionalsocialista acerca de su modelo de feminidad basado en los conceptos de maternidad y pureza racial. En segundo lugar, se estudia la adaptación de ese modelo de feminidad normativa a las políticas eugenésicas y raciales realizadas en los campos de concentración y exterminio. La investigación se ha focalizado mayoritariamente en la violencia sexual ejercida sobre las mujeres judías por la relevancia que el nacionalsocialismo concede a la «Solución Final».

Palabras clave: Feminidad normativa, Explotación Sexual, Campos de exterminio, Nacionalsocialismo. 
ABSTRACT: The article provides an overview of the sexual violence against women in the concentration and extermination camps through the testimony offered by the survivors. Women suffer a specific kind of violence with a double meaning: political and symbolic. For this reason, the sexual violence must be analyzed as a category of analysis that incorporates a gender perspective. The article is divided into two distinct and interdependent parts. First, it analyzes the nationalist discourse about the Nazi model of femininity based on the concepts of motherhood and racial purity. Secondly, it studies the adaptation of this model of normative femininity to the eugenic and racial policies carried out in the camps. The research has focused exclusively on sexual violence against Jewish women because the relevance of the "Project Final Solution" in the National Socialism politic.

Keywords: Normative Femininity, Sexual Exploitation, Extermination Camps, National Socialism.

Las investigaciones sobre las políticas de exterminio del régimen nazi han sacado a la luz las atrocidades de un proyecto político basado en los principios de superioridad racial $^{1}$, antisemitismo ${ }^{2}$ y pangermanismo ${ }^{3}$. Unos valores que se inspiran directamente en la interiorización colectiva de un sentimiento de pérdida y victimismo derivado de las condiciones de paz impuestas por el Tratado de Versalles tras el fin de la Primera Guerra Mundial. Un imaginario

1 Las doctrinas racistas esgrimidas por el nazismo constituyeron una exégesis espúrea del darwinismo en la medida en que trasladaron a las sociedades humanas las tesis acerca de la lucha por la supervivencia que Darwin había referido al mundo animal. La relación del darwinismo social y el pangermanismo nazi se sustenta ideológicamente en un libro anterior, Sobre la desigualdad de las razas bumanas de Gobineau, en el que se exalta la superioridad de la población blanca y especialmente de la aria. Posteriormente, el nazismo encumbró el tratado Los fundamentos del siglo XX de Houston Steward Chamberlain que señala a la población aria como la «única raza creadora» identificando los periodos de decadencia como resultado del mestizaje.

$2 \mathrm{El}$ antisemitismo nazi recoge una corriente de pensamiento que se venía gestando desde el siglo XIX. La cuestión judía de Dühring publicado en 1880 sostiene que la depravación es la nota definitoria del pueblo judío y que son los responsables de la degradación biológica y cultural que padece del pueblo alemán (tesis que fueron replicadas por Engels en Antidüring). Los antecedentes de las políticas de exterminio nazi ya se anuncian en la propuesta de Paasch de exterminio o deportación de los judíos a Nueva Guinea.

3 Existen muy pocas influencias pangermánicas en los círculos intelectuales románticos y liberales del siglo XIX más allá de la exaltación de la nacionalidad germana que realiza Fichte en sus Discursos a la nación alemana. Los antecedentes pangermanistas parten de la exaltación del mito Blut und Boden (Sangre y Suelo) que citará Hitler de manera recurrente. Pero las mayores influencias proceden de los Escritos alemanes de Lagarde (1881) donde se plantea la necesidad de exigir el retroceso de las fronteras rusas y de la novela Pueblo sin espacio de Hans Grimm (1926) que condensa el proyecto expansivo nazi basado en el principio de «espacio vital germano» o Lebensraum. 
colectivo en el que el pueblo alemán se reconoce humillado y sojuzgado erróneamente y que es exaltado por algunos intelectuales que predican la organización de una respuesta política. Es el caso de Spengler y su obra La decadencia de Occidente donde subraya el peligro que constituye la democracia como régimen político y ensalza la guerra como «una forma eterna de vida superior» ${ }^{4}$. En el mismo sentido se pronuncia Van der Bruck en El Segundo Reich (1923) cuando señala que «una paz ha mutilado a Alemania, nos ha robado una parte de nuestro suelo patrio, nos ha arrebatado el agua de nuestros ríos y nos ha prohibido incluso la libre disponibilidad del aire» ${ }^{5}$.

El descontento político-social y la crisis económica germana derivada de las reparaciones de guerra impuestas por el Tratado de Versalles sientan las bases de un nuevo tiempo en el que se materializa un proyecto que habría de convertir a Alemania en un nuevo referente opuesto a las demás naciones europeas. Un Tercer Reich que recogiera el legado del Sacro Imperio Romano Germánico (962-1806) y del Imperio Bismarckiano (1871-1918) y convirtiera a Alemania en un nuevo paradigma político, social, económico e ideológico. El artículo analiza en primer lugar el papel que el nuevo discurso nacionalsocialista concede a las mujeres alemanas en su nuevo proyecto de germanización y superioridad racial y, en segundo lugar, el tratamiento que reciben las mujeres en los campos de concentración y exterminio de acuerdo a su visión normativa de la feminidad y de las políticas eugenésicas y antisemitas.

\section{LA FEMinidad NORMATIVA DEL NSDAP. LA EXALTACión DE LA MATERNIDAD EN EL PROCESO DE GERMANIZACIÓN RACIAL}

Algunos de los principios sobre lo que se encumbra el proyecto nacionalsocialista del III Reich incorporan a las mujeres como el núcleo fundacional de la nueva Alemania. En ellas recae la tarea de engendrar una nueva juventud que abastezca de nuevos ideólogos al partido nazi (NSDAP) y de jóvenes soldados a la Wehrmacht ${ }^{6}$, la Schutzstaffel ${ }^{7}$ (SS),

4 SPENGLER, Oswald (2007): La decadencia de Occidente, Madrid, Espasa Calpe, Tomo 1. 5 VAN DER BRUCK (1989): El Segundo Reich, Madrid, Taurus.

6 Las nuevas fuerzas armadas unificadas de la Alemania nazi entre los años 1935 y 1945 surgidas tras la disolución de la Reichswehr, fuerzas armadas de República de Weimar.

7 Organización militar, política, policial, penitenciaria y de seguridad creada en 1925 como guardia personal Adolf Hitler. Sus orígenes se remontan al año 1923 cuando surge como 
la Sturmabteilung ${ }^{8}$ (SA) y la Gestapo'; organismos y fuerzas de control que respaldan política, social, militar e ideológicamente el proyecto del NSDAP por medio de acciones represivas y coercitivas. Las mujeres no desempeñan cargos relevantes en el proyecto del III Reich y el rol que el discurso nacionalsocialista reserva a las mujeres germanas es meramente reproductivo y asistencial de acuerdo al proyecto racial Lebensborn ${ }^{10}$ que inició Heinrich Himmler en 1935 bajo la tesis de la superioridad racial aria esgrimida por los ideólogos del NSDAP.

La exaltación de la maternidad como rasgo único y esencial de las mujeres se repite incansablemente en los discursos del NSDAP desde el momento en que los nazis alcanzan el poder en el año 1933. Las primeras iniciativas legislativas referidas a lo que el discurso nacionalsocialista denomina la «guerra de la natalidad» se centran en la creación de un sistema de préstamos estatales para parejas arias casadas en las que la mujer no trabajara fuera de casa, exenciones fiscales para los matrimonios arios que tuvieran al menos cuatro hijos, la concesión de la Cruz de Honor de la Madre Alemana a las

una compañía perteneciente a las Sturmabteilung (SA) con la función de proteger a los miembros más veteranos del NSDAP en las reuniones, discursos u otros eventos públicos de carácter nazi. Bajo el mandato de Heinrich Himmler las SS pasaron de ser una pequeña formación paramilitar a convertirse en una de las más grandes y poderosas organizaciones dentro del Tercer Reich. La Waffen-SS, el ala combatiente de las SS, evolucionó como un segundo Ejército alemán dentro de la Wehrmacht, que operaba junto al ejército regular. Bajo la dirección de Himmler las SS recibieron la autoridad de controlar el Sicherheitsdienst o Servicio de Seguridad, el Servicio de Inteligencia y Seguridad y la Gestapo o policía secreta.

8 Milicia de asalto del NSDAP. Jugaron un importante papel en el ascenso al poder del NSDAP hasta que fueron desarticuladas en 1934 e integradas en las SS.

9 Policía secreta del Estado creada en 1933 a partir de la Policía Secreta Prusiana. Bajo la total administración de la Schutzstaffel (SS), fue administrada por la Reichssicherheitshauptamt (Oficina Central de Seguridad del Reich) y considerada como una organización dual del Sicherheitsdienst (Servicio de seguridad) y una suboficina de la Sicherheitspolizei (Policía de seguridad). La función de la Gestapo era investigar y combatir «todas las tendencias peligrosas para el Estado». La Gestapo tenía autoridad para investigar los casos de traición, espionaje y sabotaje, además de los casos de ataques criminales al Estado y al NSDAP con amplios poderes ya que la ley fue modificada para que sus acciones no estuvieran sometidas a revisión judicial. Disuelta en 1945 por orden del presidente estadounidense Dwight Eisenhower.

10 Programa de reproducción selectiva destinado a promover la hegemonía de la población aria por medio de programas de bienestar social, incentivos y cuidados de excelencia en línea con las políticas raciales y eugenésicas del NSDAP. Se restringió a mujeres que fueran consideradas arias "racialmente puras" y que no estuvieran casadas con el objetivo de facilitar el nacimiento de los niños y niñas cuya adopción recaía en miembros de las SS (el acceso a las SS requería la superación de criterios raciales). 
mujeres que tenían más de cuatro hijos, la declaración del Día de la Madre en fiesta nacional y la ilegalización de los anticonceptivos para las mujeres arias «racialmente deseables». Respecto a la ilegalización del aborto para las mujeres arias Hitler declaró:

El uso de los anticonceptivos por parte de las mujeres arias significa una violación de la naturaleza, una degradación de la condición femenina, de la maternidad y del amor. Los ideales nazis exigen que la práctica del aborto sea exterminada con mano dura. Las mujeres inflamadas por la propaganda marxista reclaman el derecho a tener hijos sólo cuando lo desean. Primero pieles, muebles nuevos, luego, quizá un bijo ${ }^{11}$.

En el mismo sentido se pronuncia Joseph Goebbels ${ }^{12}$ cuando destaca la importancia de que las mujeres permanezcan en los hogares atendiendo las necesidades de los hijos por encima de cualquier necesidad vital incluyendo los ideales emancipatorios que son ridiculizados bajo la sombra de acusaciones marxistas.

Virulento debate sobre la mujer y sus tareas. En esto, soy enteramente reaccionario. Tener niños y educarlos es una gran tarea. Mi madre es la mujer a la que tengo mayor respeto, y está alejadísima del intelecto, y tanto más próxima a la vida. Hoy las mujeres opinan de todo, lo único que ya no quieren es tener hijos. Y a eso le llaman emancipación ${ }^{13}$.

Los ideales emancipatorios habían calado entre las mujeres alemanas y habían enraizado profundamente en la conciencia pública. La visibilidad de las mujeres en la esfera pública no era un «sarampión de posguerra» que podría subsanarse con los nuevos ideales nazis como proclamaba el discurso nacionalsocialista. Desde los años veinte las mujeres trabajaban en todos los sectores públicos y privados y enarbolaban los ideales emancipatorios sobre los principios del sufragismo femenino, la educación y el trabajo. En Alemania el paradigma de la «mujer moderna» que proponían los movimientos feministas de los años treinta no era sólo una iconografía simbólica sino un fenómeno social de amplio espectro. Las mujeres alemanas no sólo

11 KOONZ, Claudia (2005): La conciencia naz̧. La formación del fundamentalismo étnico del III Reich, Barcelona, Paidós.

12 Ministro de Propaganda e Información del Reich desde 1933 y sucesor de Hitler en la Cancillería del Estado.

13 KOONZ, Claudia. Ibid. 
reivindicaban un espacio propio en el ámbito público sino que además irrumpían con fuerza en ámbitos tradicionalmente masculinos. Las mujeres ocupaban puestos académicos en las universidades, irrumpían con fuerza en el ámbito político e incluso se celebraban carreras de coches conducidos por mujeres y se organizaban actos deportivos en los que mostraban sus capacidades como aviadoras y paracaidistas como es el caso de Melitta Schiller, doctora en Ciencias Físicas y piloto de pruebas de aviación que realizó más de 1500 vuelos experimentales hasta que fue expulsada de la Luftwaffe por sus orígenes judíos ${ }^{14}$.

Desde años los veinte surgen nuevos modelos de feminidad que entran en conflicto con el ideal nacionalsocialista de «la mujer junto a la rueca y el costurero» que, paradójicamente, no era asumido por las mujeres cercanas a la jerarquía nazi que eran expuestas como modelos de la nueva germanización nacionalsocialista. Eva Braun, compañera de Adolf Hitler, siempre mostró una actitud frívola y burguesa, Carin y Emmy Göring, primera y segunda esposa de Hermann Göring ${ }^{15}$, se abrieron paso como agitadora política y actriz de cine respectivamente, Henriette von Schirach, hija del fotógrafo Heinrich Hoffmann ${ }^{16}$, activó la vida cultural vienesa y Leni Riefenstahl se convirtió en la «cineasta de Hitler» con sus filmaciones megalómanas de los mítines nazis. Tampoco interiorizaron las doctrinas de la reproducción y la maternidad esgrimidas por la propaganda nacionalsocialista ya que sólo las familias Bormann y Goebbels respondieron al ideal familiar nazi basado en los conceptos de germanización y superioridad racial. Gerda Bormann y Magda Goebbels, esposas del secretario del partido NSDAP y del ministro de propaganda respectivamente, eran las únicas mujeres del círculo de jerarcas nazis que respondían al nuevo paradigma de feminidad con la crianza de numerosos hijos e hijas y una vida doméstica basada en la fidelidad al marido y a la causa nacionalsocialista.

La creación de la Liga de Muchachas Alemanas (BDM) fundada en 1930 como la rama femenina de las Juventudes Hitlerianas (HJ) para chicas de entre 10 y 18 años fue el proyecto más ambicioso del NSDAP para divulgar y naturalizar los nuevos ideales de feminidad. Hasta que los nazis llegaron al poder esta organización no tuvo una relevancia social destacable pero desde

14 BRACKE, Gerhard (2003): Melitta Gräfin Stauffenberg. La vida de una aviadora, Frankfurt, Ullstein.

15 Comandante supremo de la Luftwaffe y Mariscal del Reich.

16 Fotógrafo personal de Hitler y militante del NSDAP desde 1920. 
1933 creció rápidamente hasta que el ingreso se tornó obligatorio en 1936 para todas aquellas jóvenes alemanas, arias y libres de enfermedades hereditarias. Las chicas eran educadas y socializadas en un entorno nacionalsocialista que les preparaba para el desempeño de los roles asistenciales y maternales impulsados por el NSDAP y cuyo adoctrinamiento comprendía el traslado temporal a granjas de familias numerosas para conseguir «la emancipación de la emancipación de la mujer» que recordaba insistentemente Alfred Rosenberg ${ }^{17}$. Durante los últimos meses de la Segunda Guerra Mundial, muchas jóvenes de la BDM participaron activamente en la defensa de Berlín ante el avance de las tropas aliadas. No se puede saber el alcance de su participación en la defensa de la ciudad ya que no tomaron parte de manera conjunta y coordinada sino que combatían de manera voluntaria junto a las HJ y a la Wehrmacht.

El NSDAP discriminó a las mujeres sobre una ideología misógina erigida sobre los principios de maternidad, germanización y superioridad racial. La proyección de un modelo de feminidad normativa centrada exclusivamente en la capacidad reproductiva de las mujeres alemanas determina el posterior tratamiento que reciben las mujeres en la denominada «cuestión judía». Si el valor de las mujeres radica en su vertiente procreadora, ¿qué percepción social se proyecta de las mujeres judías en un contexto determinado por el exterminio sistemático del pueblo judío?

\section{Mujeres y Genocidio. La desexualización y explotación SEXual de LAS MUjeres JUdías en la SOluCión Final}

Aunque la aceptación germana de las ideas antisemitas se había ido desarrollando desde finales del siglo XIX y la discriminación efectiva se impulsa desde el ascenso del NSDAP, la decisión de afrontar el exterminio del pueblo judío fue tomada entre finales del verano y principios del otoño

17 Comisario para la Supervisión de la Educación Intelectual e Ideológica del NSDAP desde 1934 y Ministro del Reich para los Territorios ocupados del Este desde 1941. Máximo impulsor de las tesis de la superioridad racial y de la Solución Final en el III Reich. Su ideario antisemita le llevó a crear el Instituto para el Estudio de la Cuestión Judía dedicado a identificar y atacar la influencia judía en la cultura alemana y registrar la historia del judaísmo desde una perspectiva antisemita en el libro El mito del siglo XX donde expone su teoría racial basada en la hegemonía de la población aria. 
del año $1941^{18}$ y emergió en su plenitud en la primavera de $1942^{19}$ de la mano de Heinrich Himmler ${ }^{20}$. El exterminio sistemático conocido como Solución Final pretendía poner fin a la denominada «cuestión judía» que el discurso nacionalsocialista definió como el lastre y degeneración moral y económica que la influencia semita insuflaba a la cultura, sociedad y economía germana. La «cuestión judía» se perfila retórica e ideológicamente como «una lucha a vida o muerte entre la raza aria y el bacilo judío» y exaltaba la efectividad del NSDAP «para emprender una solución final de la cuestión» de la que Hitler apenas se hizo eco públicamente pero que anunció de manera elocuente en el discurso pronunciado el 30 de enero de 1939 en el Parlamento alemán.

Durante la época de mi lucha por el poder, fue en primer lugar la raza judia la que no bižo sino recibir a carcajadas mis profecias cuando dije que algín día asumiría la dirección del Estado y, con ella, la de toda la nación, y que entonces, entre muchas otras cosas, resolvería el problema judio. Sus carcajadas fueron escandalosas, pero creo que, de un tiempo a esta parte, ya solo rien por dentro. Hoy seré profeta una vez. más: si los financieros judios internacionales de Europa y de fuera de ella logran sumir de nuevo a las naciones en una guerra mundial, el resultado no será la bolchevización de la tierra y, por lo tanto, la victoria de los judios, sino la aniquilación de la raza judía en Europa ${ }^{21}$.

Las Leyes de Núremberg constituyen el corpus legislativo que, adoptado por unanimidad el 15 de septiembre de 1935 durante el séptimo congreso anual del NSDAP, sentó las bases de la legislación sobre la pureza racial. El objetivo principal no era tanto la prohibición de las creencias religiosas judías sino evitar la mezcla racial y cultural con la población germana. La «Ley para la protección de la sangre y el honor alemanes» integrada en dicho corpus legislativo contenía en su primera disposición la prohibición expresa de toda unión entre alemanes y judíos ya sea por matrimonio, cohabitación o relación sexual. Por otra parte la «Ley de la ciudadanía del Reich» establecía una división entre alemanes y judíos al consagrar a los primeros como

18 KERSHAW, Ian (2009): «¿Genocidio improvisado? La aparición de la Solución Final en el Warthegau», en Hitler, los alemanes y la Solución Final, Madrid, La Esfera de los Libros, pág. 151.

19 Kershaw, Ian. Ibid. pág. 431.

20 Comandante en jefe de las SS desde 1929, Ministro del Interior del Reich desde 1943, Comandante de los ejércitos del Vístula durante el sitio de Berlín e ideólogo y organizador de la Solución Final.

21 WISTRICH, Robert S. (2002): Hitler y el Holocausto, Barcelona, Mondadori. pág. 122. 
«ciudadanos del Reich» y reducir a los segundos a la categoría de «nacionales o residentes» sin pertenencia al Reich lo que les privaba de los derechos civiles inherentes a la ciudadanía facilitando así la discriminación progresiva de la que fueron objeto hasta su definitiva deportación a los campos de concentración y exterminio.

Tras la celebración de la Conferencia de Wannsee ${ }^{22}$ el 20 de enero de 1942 y la posterior puesta en marcha de la Operación Reinhard ${ }^{23}$ se inicia el traslado masivo de población judía a los campos de concentración y exterminio para liberar espacio en los ghettos ante las nuevas deportaciones de judíos procedentes de los territorios ocupados en Europa occidental ${ }^{24}$. A continuación se analiza exclusivamente la percepción social que el NSDAP ofrece de las mujeres judías y la explotación sexual que padecen tras su deportación a los campos de concentración y exterminio.

Las mujeres judías se convierten en el núcleo simbólico sobre el que se edifica el discurso ligado al proyecto de la Solución Final. El NSDAP identifica a las mujeres con la capacidad de reproducción y perpetuación de las poblaciones por lo que el proyecto de exterminio del pueblo judío requiere una retórica que deslegitime no sólo a la totalidad de la comunidad judía sino específicamente a las mujeres. La deshumanización que padecen las mujeres judías en el discurso nacionalsocialista se materializa en el tratamiento violento y vejatorio que reciben en los campos de concentración y exterminio y que convierte el cuerpo femenino en un espacio simbólico de conquista y dominación.

Las agresiones, sean o no de carácter sexual, son una proyección androcéntrica de la superioridad de los vencedores sobre los vencidos a

22 Reunión de los máximos representantes del gobierno alemán en la que se debate la necesidad de implementar y coordinar la Solución Final diseñada por Reinhard Heydrich y Adolf Eichmann a través de las deportaciones masivas de la población judía a los campos de concentración del Este y su posterior exterminio. Los criterios que determinan quiénes eran o no judíos se determinaban según las cláusulas de las Leyes de Nuremberg.

23 Operación diseñada por Reinard Heydrich que materializa en 1942 el proyecto de exterminio acordado en la Conferencia de Wannsee. La operación se inicia con la creación en Polonia de los campos de exterminio de Chelmno, Treblinka, Sobibor, Belzec y posteriormente Auschwitz-Birkenau donde se inician los asesinatos masivos y sistemáticos en cámaras de gas sustituyendo así las actividades de los Einsatzgruppen («equipos móviles de matanza») que actuaban en los territorios ocupados de Europa Oriental.

24 HILBERG, Raul (2003): The Destruction of the European Jews. New Haven, CT: Yale University Press. 
través de la apropiación del cuerpo femenino. La violencia, rasgo esencial que históricamente ha definido la categoría de lo masculino, alcanza su mayor significación en los conflictos bélicos entendidos como la mayor expresión de violencia. La demostración de la agresividad y «superioridad moral» de los ejércitos vencedores requiere el despliegue de actos violentos que subrayen su masculinidad, virilidad y capacidad de conquista; una proyección extrema del androcentrismo que atenta contra el cuerpo femenino. La diferencia fundamental de las agresiones contra las mujeres en tiempos de paz y en tiempos de guerra es inapreciable para las víctimas pero nítida para los agresores. Frente la reivindicación de la dominación sobre el cuerpo de las mujeres que se deriva de las violaciones acaecidas en tiempos paz, las violaciones producidas en conflictos bélicos perfilan una doble significación: la dominación sobre el cuerpo femenino y la proyección de las mujeres como una posesión masculina mediante la que deslegitimar y humillar a los enemigos; las mujeres se convierten en proyecciones de los ejércitos vencidos y sus cuerpos en nuevos espacios de conquista.

La proyección del cuerpo femenino como un espacio de conquista y dominación constituye la base ideológica sobre la que el discurso del NSDAP legitima las agresiones contra las mujeres; unas agresiones en las que la sexualidad siempre está presente como se aprecia en los testimonios ofrecidos por las supervivientes de los campos de Chelmno ${ }^{25}$ AuschwitzBirkenau ${ }^{26}$, Bergen-Belsen ${ }^{27}$ y Ravensbrück ${ }^{28}$ recogidos en el proyecto USC

25 Primer campo de exterminio creado en la Operación Reinhard. Construido originariamente como un proyecto piloto de los campos de Trebinkla, Sobibor y Belzec.

26 Uno de los principales campos de concentración, experimentación y exterminio. Inaugurado en 1939 bajo la dirección de Heinrich Himmler tras la invasión de Polonia para alojar a los judíos deportados de Europa del Este. Tuvo tres ampliaciones de las cuales Auschwitz II Birkenau fue destinada al exterminio masivo de la población judía. Fueron asesinados entre 1,5 y 3 millones de prisioneros.

27 Campo de trabajo construido en 1936 para albergar a los 3.000 trabajadores que habían de edificar los cuarteles acorazados de Bergen. En 1939 se convierte en campo de prisioneros de guerra y en 1943 pasa a ser controlado por las SS y convertido en campo de concentración para alojar a los judíos deportados de Europa del Oeste. Ingresaron aproximadamente 95.000 prisioneros la mayoría de los cuales perecieron a causa de la malnutrición y las enfermedades endémicas.

28 Campo de concentración de mujeres inaugurado en 1939 por orden de Heinrich Himmler. En 1941 se incorporó un pequeño campo adyacente para hombres y en 1942 se añadió el llamado "Campo preventivo de menores de Uckermark" para jóvenes y niñas. Entre 1939 y 1945 ingresaron como prisioneros alrededor de 132.000 mujeres y niños, 20.000 hombres y 1.000 chicas adolescentes. Entre 6.000 y 10.000 mujeres perecieron en las cámaras de gas. 
Shoab Foundation Institute for Visual History and Education ${ }^{29} \mathrm{y}$ en los archivos históricos del Center for Advanced Holocaust Studies ${ }^{30}$ dependiente del United States Holocaust Memorial Museum (USHMM) cuyos archivos históricos constituyen la principal fuente documental del artículo.

La jerarquización racial inherente a la ideología nacionalsocialista se materializa en la separación física de los prisioneros de los campos de exterminio según su asignación racial y su sexo. Las mujeres judías ocupaban la posición más baja en la jerarquización piramidal de los prisioneros por su doble condición de mujeres y judías. Su doble discriminación les aleja de las actividades más sencillas o menos agotadoras y se ven obligadas a desempeñar los trabajos más duros y minuciosos dentro de la actividad diaria de los campos. Su estatus en el escalafón más bajo convierte a las mujeres judías en el principal objetivo de la violencia sexual ejercida no sólo por los guardas de las SS sino también por los propios prisioneros judíos. En los campos de concentración y exterminio en los que no se aplicaba la segregación por sexos se han documentado actos de agresión sexual por parte de los propios prisioneros.

Todas las mujeres padecieron en mayor o menos medida la explotación sexual por parte de las autoridades de las SS que custodiaban los campos de exterminio aunque el artículo se centra fundamentalmente en la violencia ejercida contra las mujeres judías. Se han documentado numerosos testimonios que recogen el trato que recibían las mujeres desde su entrada en los campos de concentración y exterminio. Se han seleccionado los testimonios más completos para ilustrar la naturaleza y significado de las agresiones y explotación sexual que, en virtud del discurso racial que prohibía las relaciones con las mujeres judías, no tenía tanto un matiz sexual como exclusivamente denigratorio.

Los primeros actos de agresión no tenían un carácter específicamente físico sino psicológico tendente a minar la percepción que tenían las mujeres sobre sí mismas y desencadenar un proceso de desexualización

29 Organización sin ánimo de lucro creada por Steven Spielberg en 1994 con el objetivo de recoger los testimonios de los supervivientes del Holocausto mediante entrevistas filmadas. La mayor parte de sus grabaciones fílmicas y sonoras se han integrado en el Archivo Histórico del USHMN.

30 Centro de estudios creado en 1998 para impulsar nuevos estudios sobre el Holocausto. Depende del United States Holocaust Memorial Museum (USHMM) y cuenta con un extenso archivo histórico dirigido por el Holocaust Survivors and Victims Resource Center. 
y deshumanización gradual. El primer paso de deconstrucción del sujeto femenino afectaba a las madres quienes eran despojadas de sus hijos para fragmentar de manera irreversible su actividad maternal. La separación física de las madres de sus hijos e hijas desarticula el núcleo familiar de las mujeres y les arrebata la función que el discurso nacionalsocialista considera el núcleo de su feminidad normativa: la maternidad. Helen Lebowitz, superviviente del campo de Bergen-Belsen, describe la separación de las madres y sus hijos e hijas y las consiguientes agresiones físicas padecidas a manos de los guardas de las SS.

Recuerdo a esas madres que bajaban con niños pequeños, y ellos... ellos trataban de arrebatárselos de las manos. Sabes que es muy dificil separar a una familia. Es muy dificil. La gente se resiste. Habia muchos gritos. Recuerdo el camión. Las madres que no renunciaban a los niños fueron golpeadas y los niños heridos. Cogieron a los niños y los arrojaron al camión sin prestarles atención. En ese momento vimos que algo horrible estaba sucediendo -la forma en que se comportaban con los niños y los bebés. En el camión habia gente en muy malas condiciones, ya sabes, estaban arrojando alli a los enfermos y... y... y estos niños pasaron un momento muy difícil. Fueron lanzados a los camiones. Y habia tantas madres que estaban corriendo tras los camiones que, por supuesto, las golpearon y empujaron bacia atrás ${ }^{31}$.

Las mujeres, una vez separadas de sus hijos e hijas, sufrían un proceso selectivo que de acuerdo a sus capacidades físicas determinaba su validez para el trabajo requerido en los campos. Las mujeres eran sometidas a un proceso de homogeneización física que bajo la retórica de la higiene convertía a todas las mujeres en sujetos desindividualizados. Las mujeres eran trasladadas a barracones en los que se inspeccionaba detenidamente su cuerpo, se les cortaba el cabello y se les rasuraban sus partes íntimas. La degradación que suponía ser examinadas íntimamente por las trabajadoras del campo se recoge en numerosos testimonios como el aportado por Micheline Maurel en el que relata su llegada al campo de Ravensbrück.

Nos pusieron en fila y nos hacian entran en pequeños grupos en un barracón donde nos obligaban a desnudarnos a toda prisa y desgarraban la ropa de las mujeres que se negaban a desnudarse... nos tendían en una mesa

31 Grabación fílmica del Archivo Visual del United States Holocaust Memorial Museum. Story Number RG. 60-6654. Tape Number: 6534-9747. 
de metal, fría como el bielo, e inspeccionaban todos los orificios naturales del cuerpo... era una bumillación absoluta ${ }^{32}$.

Las mujeres experimentaban no sólo la humillación de los exámenes físicos sino que además eran golpeadas sistemáticamente para comprobar su fortaleza física. Sarah y Esther Hoffmann recuerdan que las mujeres eran golpeadas en las nalgas mientras desfilaban desnudas durante el proceso de selección en el campo de Auschwitz-Birkenau ${ }^{33}$. Pese a la humillación que suponían tales exámenes físicos, la mayoría de los testimonios inciden en que el punto de inflexión que les hizo ser conscientes de la deshumanización y desexualización a la que iban a ser sometidas se concreta en el corte de cabello. Las supervivientes perciben ese acto como un episodio en el que no sólo sesgan su individualidad sino que mutilan su feminidad. Cuando las supervivientes describen su apariencia física sin cabello se refieren así mismas como personas indistinguibles unas de otras, «masas monolíticas» ${ }^{34}$, «animales» $»^{35}$ e incluso «subhumanas» ${ }^{36}$. Anna Friedman, superviviente de Ravensbrück, declara que «pensábamos que éramos como animales, ¿quién había visto antes a una mujer sin cabello? $\rangle^{37}$. En este sentido se pronuncia Martha Michelsohn, superviviente del campo de Chelmno.

Yo no quería perder mi cabello. Eso es lo peor que le puede pasar a una mujer $[. .$.$] te arrebatan aquello que te distingue y te hace una$ mujer. Es un momento terrible. Es como si me bubieran quitado la piel, como si se bubiera volatilizado la personalidad. Se veía a las mujeres sin cabello, con las orejas prominentes y los ojos hundidos y asustados, era algo salido de una pesadilla ${ }^{38}$.

32 Grabación fílmica del Archivo Visual del United States Holocaust Memorial Museum. Story Number RG. 60-8790. Tape Number: 0098-3212.

33 Campo de concentración y exterminio inaugurado en 1939 cerca de Cracovia. Dirigido por Rudolf Hess hasta 1943 se convirtió en el núcleo central del proyecto «Solución Final». Integrado por tres campos: Auschwitz I, Auschwitz II Birkenau y Auschwitz III. Las mujeres fueron recluídas en Auschwitz II Birkenau y desde 1942 se convirtió en el principal campo de exterminio.

34 Grabación fílmica del Archivo Visual del United States Holocaust Memorial Museum. Story Number RG. 60-9876. Tape Number: 9865-7347.

35 Grabación fílmica del Archivo Visual del United States Holocaust Memorial Museum. Story Number RG. 60-8965. Tape Number: 8653-0864.

36 Grabación fílmica del Archivo Visual del United States Holocaust Memorial Museum. Story Number RG. 60-8754. Tape Number: 8653-9864.

37 Grabación fílmica del Archivo Visual del United States Holocaust Memorial Museum. Story Number RG. 60-8641. Tape Number: 0964-8070.

38 Grabación fílmica del Archivo Visual del United States Holocaust Memorial Museum. 
El impacto psicológico de la pérdida del cabello ocasiona una sensación de inseguridad y vergüenza que se incrementa con la presencia de miembros de las SS durante el corte del cabello y el rasuramiento de la zona púbica. Libuse Nachtmanova, superviviente del campo de Treblinka, describe que cuando los hombres de las SS entraban en la zona de las duchas las mujeres no se cubrían los pechos o la zona púbica con las manos como sería de esperar. Las mujeres se cubrían la cabeza porque «para ellas era más vergonzosa su cabeza rasurada que su desnudez corporal ${ }^{39}$. Las mujeres interpretan el corte del cabello como una violación de su integridad corporal y una negación de su identidad individual.

Debido a las situaciones de estrés y a la posterior malnutrición y explotación física muchas mujeres desarrollaron amenorrea o ausencia temporal de la menstruación. La amenorrea no fue causada intencionalmente por las autoridades médicas de los campos de concentración y exterminio sino que responde a un mecanismo natural de defensa que padecen las mujeres en situaciones en las que la reproducción es inviable debido a cuestiones biológicas (escasa nutrición, pérdida drástica de defensas, estrés etc.). Esta situación agrava la percepción subjetiva de las mujeres y agudiza el proceso de desexualización como subraya Lucille Engel, superviviente del campo de Bergen-Belsen.

Era tremendamente doloroso... padecía temblores y dolores muy agudos en los ovarios, tenía calambres y lo peor es que dejé de sentirme una mujer, me vi a mi misma como una anciana, estérily enferma en un cuerpo que ya no reconocia ${ }^{40}$.

La idea de que habían dejado de «ser mujeres» se repite en muchos de los testimonios recogidos. Las autoridades médicas no aplicaron tratamientos médicos tendentes a paliar esta situación ya que la amenorrea facilitaba la esterilidad y frenaba por tanto los posibles embarazos que pudieran gestarse durante la estancia de las mujeres en los campos. No obstante, pese al proceso de desexualización sí se fomenta la visibilidad de la sangre menstrual en aquellas mujeres que tardaron más tiempo en padecer la amenorrea. Hannah

Story Number RG. 60-3843. Tape Number: 3947-4823.

39 Grabación fílmica del Archivo Visual del United States Holocaust Memorial Museum. Story Number RG. 60-9087. Tape Number: 9800-0086.

40 Grabación fílmica del Archivo Visual del United States Holocaust Memorial Museum. Story Number RG. 60-6533. Tape Number: 9800-6411. 
Kryshak, superviviente de Ravensbrück, relata el acoso que padecían las mujeres menstruantes a las que se obligaba a ir desnudas.

Las pocas mujeres que continuaban menstruando eran obligadas a ir desnudas para que la sangre fuera visible, disfrutaban viendo la sangre deslizarse por las piernas como si fuéramos animales... y después les obligaban a limpiar las manchas que dejaban en el suelo de los barracones... era una denigración ${ }^{41}$.

Esta situación crea una paradoja que bascula entre la preocupación de las mujeres por su deterioro físico, del que la amenorrea era una muestra evidente, y el agravio y humillación que padecían si continuaban menstruando. Se crea un conflicto psicológico en el que su cuerpo entra en pugna con la violencia cotidiana que se desarrolla en los campos de concentración y exterminio. El deseo de mantener intacta su salud rivaliza frontalmente con el deseo de padecer amenorrea para paliar las humillaciones y evitar posibles embarazos resultado de las violaciones.

La esterilización forzada, en el nombre de experimentos pseudocientíficos, es una forma más severa e irreversible de violencia sexual que se ejerció sobre miles de mujeres. La mutilación del cuerpo femenino constituye la máxima representación de la denigración a las que fueron sometidas las mujeres porque manipula y modifica su sexualidad para satisfacer un proyecto político e ideológico con unos objetivos genocidas. El cuerpo de las mujeres se proyecta como un espacio físico y simbólico, inerte y objetivado, que puede transformarse desde una significación física y política. Bajo el liderazgo e iniciativa de Heinrich Himmler, a partir del año 1941 las SS incorporan a los campos tratamientos médicos de esterilización para experimentar y frenar la reproducción de la forma más rápida y efectiva posible. Tales tratamientos se dirigen exclusivamente a las mujeres, e incluso a niñas que aún no han llegado a la madurez sexual, por medio de operaciones quirúrgicas, radiaciones e inyecciones hormonales que se configuran como estrategias de ejecución del proyecto «Solución Final». Anja Lundholm informó de miles de esterilizaciones diarias en Auschwitz-Birkenau que se realizaban sin anestesia y que se cobraron cientos de vidas de mujeres y niñas que no sobrevivieron a las intervenciones ${ }^{42}$.

41 Grabación fílmica del Archivo Visual del United States Holocaust Memorial Museum. Story Number RG. 60-0912. Tape Number: 9800-1204.

42 LUNDHOLM, Anja (1988): The gate to hell. Report of a survivor, Rowohlt, Reinbecj debi 
Uno de los medios más utilizados para extender la esterilización masiva entre las mujeres judías se basaba en la ingesta de productos químicos altamente tóxicos diluidos en las raciones alimentarias. Los efectos de tales ingestas eran visibles y sus consecuencias incluían dolores intensos, hemorragias internas, perforaciones de la cavidad bucal, atrofia de los ovarios y una amenorrea irreversible. Janny Brandes-Brilleslijper, superviviente del campo de exterminio de Auschwitz-Birkenau, recuerda cómo el temor a ingerir productos tóxicos llevó a muchas mujeres a no ingerir alimentos acelerando así el proceso de desnutrición.

Durante todos los años que estuvimos internas en el campo de Auschwitz-Birkenau temíamos que nos mezclaran veneno en la comida... era una situación enloquecedora... teniamos todos los sentidos alerta... sabiamos que muchas mujeres enfermaban a causa del mal estado de los alimentos y temíamos que nos estuvieran envenenando... corrian muchos rumores y se decía que nos estaban esterilizando poco apoco ${ }^{43}$.

El temor a la esterilización impulsó a muchas mujeres a renunciar a la comida deteriorando gravemente su salud física y poniendo en riesgo su valor como mano de obra. El cuidado físico constituyó una preocupación vital para las mujeres judías ya que si menguaba su capacidad de trabajo eran susceptibles de ser eliminadas en las cámaras de gas. Las mujeres sólo tenían valor en la medida en que eran una fuente de trabajo barata y eficaz por lo que debían mantener en la medida de lo posible su fortaleza física. Sin embargo, el temor a ser esterilizadas a través de un envenenamiento progresivo desencadenó y aceleró procesos de malnutrición. Las mujeres se vieron obligadas a decidir entre el mantenimiento de su fertilidad, ya temporalmente menguada debido al desarrollo de la amenorrea, o a ingerir alimentos para garantizar su permanencia en los barracones de trabajo. Incluso tras el desmantelamiento de los campos de exterminio y la consiguiente liberación de las prisioneras, se mantuvo el temor a la infertilidad y a los efectos de los tratamientos médicos recibidos durante el tiempo de cautiverio como relata Anna Stern, superviviente de Ravensbrück.

Hamburg, pp. 182-183.

43 Grabación fílmica del Archivo Visual del United States Holocaust Memorial Museum. Story Number RG. 60-7643. Tape Number: 0864-9935. 
Después de que terminara la guerra la mayoría de nosotras nos moriamos de miedo ante la posibilidad de quedarnos embarazadas. Queríamos quedarnos embarazadas para comprobar si nos habiamos quedado estériles a consecuencia de la malnutrición, el trabajo forzado y los tratamientos de esterilización que padecimos en Ravensbrück, pero al mismo tiempo nos aterrorizaba la posibilidad de que nuestros bijos nacieron enfermos o con malformaciones debido a los tratamientos recibidos ${ }^{44}$.

Las intervenciones quirúrgicas y las radiaciones para extirpar o dañar irreversiblemente los ovarios fueron la segunda medida de esterilización diseñada por las autoridades médicas de los campos de concentración y exterminio. En el campo de Dachau se realizaron miles de esterilizaciones quirúrgicas para la educación de estudiantes de medicina trasladados directamente desde Berlín. Ceijka Stoijka, superviviente de Ravensbrück, señala los efectos de tales intervenciones.

Desde los barracones anexos se oían los gritos de las mujeres que eran intervenidas. Les abrían en canal y les quemaban los ovarios con rayos $X o$ se los extirpaban... sin anestesia... con plena consciencia para ver hasta qué punto eran capaces de aguantar el dolor. Vi a niñas de ocho y nueve años que salían gritando de los barracones médicos, empapadas en sangre y en sudor, deambulando sin sentido por las calles y gritando a causa del dolor... les habian extirpado los ovarios ${ }^{45}$.

Junto a los procesos de esterilización, los abortos forzados constituyeron la segunda estrategia de deslegitimación de las mujeres al invadir el cuerpo femenino y coartar cualquier posibilidad reproductiva en virtud de una ideología basada en el principio de la superioridad racial. La ideología nacionalsocialista coacciona la reproducción de las «razas inferiores» dentro y fuera de los campos de concentración y exterminio. Las intervenciones abortivas, mientras que eran una actividad prohibida y castigada con la pena de muerte para todas las mujeres arias y libres de enfermedades hereditarias, constituyeron una actividad rutinaria en los campos para frenar los efectos de las violaciones y no devaluar la fuerza de trabajo de las mujeres; los

44 Grabación fílmica del Archivo Visual del United States Holocaust Memorial Museum. Story Number RG. 60-8642. Tape Number: 8631-9806.

45 Grabación fílmica del Archivo Visual del United States Holocaust Memorial Museum. Story Number RG. 60-7465. Tape Number: 0670-9744. 
abortos se incrementaron notablemente tras la instauración de barracones destinados específicamente a la explotación sexual (burdeles o Sonderbauten) como se detalla más adelante.

La incertidumbre que padecen las mujeres ante la posibilidad de padecer violaciones sexuales ejercía una presión adicional al estrés psicológico porque incorpora comportamientos sexistas y sexuales en la vida rutinaria de los campos de exterminio y crean una aparente contradicción: frente a la desindividualización y desexualización derivada del trato que reciben por parte de las autoridades de las SS que mutila la subjetividad y el cuerpo de las mujeres, se mantienen actitudes que las sexualizan y les hace conscientes de su categorización como objetos sexuales. Las leyes de Nüremberg prohibían las relaciones sexuales entre los alemanes y las mujeres judías sin embargo, aunque esta restricción se mantuvo entre los altos mandos de las SS que dirigían los campos de concentración exterminio, se registran casos de abusos sexuales y violaciones por parte sobretodo de los mandos intermedios de las SS y de prisioneros de alto rango.

En algunas ocasiones la aceptación de los abusos sexuales ejercidos por las autoridades de las SS constituían una garantía de supervivencia para las mujeres judías más jóvenes. Las adolescentes podían obtener mayores garantías de acceder a comida, ropa u objetos de higiene con mayor facilidad que las demás mujeres a través de los favores sexuales con miembros de las SS o con prisioneros de alto rango. Sin embargo, la sensación de seguridad que se derivan de tales relaciones se matiza con el peligro que suponía que tales relaciones se hicieran públicas ya que esas conductas eran reprobadas por las prisioneras y prisioneros de los campos. La percepción del sexo como un instrumento de supervivencia debe ser analizada como una forma de coerción sexual en la medida en que sólo son consentidas para paliar otras formas de violencia. Irena Liebman describe la aceptación de los abusos sexuales como garantía de supervivencia en una escena que presenció a su llegada al campo de exterminio de Mauthausen.

Estábamos sentadas en el suelo, esperando a que nos llevaran a los barracones. Uno de los criminales de las SS vino hacia nosotras con dos latas de sardinas en la mano. Se acercó a una joven y copularon a la vista de todos... Él le dio las dos latas de sardinas. Fue la primera vez que vi algo así, ¿sabes a lo que me refiero? ?t $^{40}$

46 Grabación fílmica del Archivo Visual del United States Holocaust Memorial Museum. 
Los abusos sexuales eran una amenaza constante y muchas mujeres deforman la percepción de su propio cuerpo para evitar despertar la atención de los guardas de las SS. El ocultamiento del cuerpo, el envejecimiento de su aspecto y la agudización consciente de las consecuencias físicas derivadas de las condiciones de malnutrición, hacinamiento y trabajo forzado se convierten en estrategias de supervivencia o resistencia sexual que en última instancia deforman las identidades de las mujeres y su sexualidad. Las mujeres se ven obligadas a renunciar y ocultar su cuerpo, a ser descorporeizadas e invisibilizadas, a ser víctimas silenciosas para no despertar la agresividad sexual.

La mayor expresión de la violencia y explotación sexual que padecen las mujeres en los campos de concentración y exterminio es la creación de los Sonderbauten o burdeles dirigidos a satisfacer las necesidades sexuales de los prisioneros de alto rango. Los primeros estudios sobre los Sonderbauten surgen a mediados de los años noventa gracias a las investigaciones de Christa Schult ${ }^{47}$. Hasta ese momento, y pese a los testimonios de las supervivientes, la noción de los burdeles en los campos de exterminio se percibió como un fenómeno aislado que se diluyó bajo otras formas de explotación sexual. El carácter específico de la violencia sexual que conlleva la prostitución forzada requiere un análisis más detallado.

El primer Sonderbauten se crea en el campo de Mauthausen en julio de $1942^{48}$. La elección de Mauthausen no es una decisión casual e ilustra los argumentos justificativos del NSDAP para extender los Sonderbauten a otros campos de exterminio. Mauthausen se edificó junto a una cantera de granito de la que se extraía el material para la construcción de los grandes proyectos arquitectónicos de Berlín, Núremberg, Hamburgo y Munich. Heinrich Himmler adopta la decisión de habilitar un burdel en Mauthausen tras una visita al campo y a la cantera cercana en 1941. En este sentido, y como señala Robert Sommer ${ }^{49}$, una justificación plausible de tal decisión pudo ser la necesidad de acelerar la extracción de granito y por tanto la

Story Number RG. 60-7853. Tape Number: 0086-8400.

47 SCHULZ, Christa (1994): «Female prisioners from Ravesnbrück in brothels of concentrations camps for males», Claus Fullberg (coord.): Women in concentration camps, Bremen, Editions Temmen.

48 Archivos del Mauthausen Memorial, Bodellbuch Block 3. AMM K2-1.

49 SOMMER, Robert (2010): «Sexual Explotaiton of women in nazi concentration camp brothels», Hedgepeth, S \& Saidel R. (eds): Sexual violence against Jewish women during the Holocaust, Lebanon, Brandeis University Press. 
productividad del campo garantizando a los prisioneros más eficientes el derecho a mantener relaciones sexuales a cambio del pago de una cantidad económica que se realiza en forma de cupones. No obstante, con el tiempo los propios guardianes de las SS mantuvieron relaciones sexuales con las mujeres de los Sonderbauten. A mediados de 1943 la construcción de los Sonderbauten se convirtió en una estrategia para implementar la productividad de los internos en los trabajos forzosos que realizaban en los campos. La aceptación de los burdeles como parte de un sistema de incentivos dio a la cotidianeidad de los campos una nueva dimensión: la explotación sexual de las mujeres de forma sistemática como un incentivo y un privilegio para los prisioneros.

La selección de las mujeres que debían ejercer la prostitución en los Sonderbauten se realizaba fundamentalmente entre las prisioneras del campo de Ravensbrück y en menor medida de Auschwitz-Birkenau. Las SS reclutaban a las mujeres a través de diversas estrategias selectivas. En general, los oficiales se dirigían a las prisioneras más jóvenes y sanas que trabajaban en los Kommandos o escuadrones de trabajo más duros como los dedicados a la construcción con la falsa promesa de que podrían abandonar los campos en seis meses. Esta oferta se realizaba especialmente a las jóvenes alemanas confinadas en los campos por conductas «asociales» entre las que se incluía la disidencia política, una orientación sexual lésbica y el haber ejercido la prostitución.

Las mujeres aceptaban la explotación sexual bajo una falsa promesa que no sólo conllevaba la prostitución de su cuerpo sino que además generaba traumas psíquicos en un doble sentido: el sentimiento de vergüenza por aceptar la prostitución y el sentimiento de culpa y humillación por haber aceptado una situación de dominio bajo un engaño ya que a los seis meses volvían a los campos en los que habían sido reclutadas. Los efectos psicológicos aceleraron el proceso de desindividualización de las mujeres y muchas de ellas propiciaron su muerte. Las mujeres prestaban y perdían el control de sus cuerpos como señala Ruth Elías, superviviente de AuschwitzBirkenau, cuando describe a las mujeres que regresaban de los Sonderbauten como «criaturas lamentables con un aspecto espantoso e indescriptible» ${ }^{50}$. Olga Lotar, prisionera política de Ravensbrück, recuerda el proceso de

50 Grabación fílmica del Archivo Visual del United States Holocaust Memorial Museum. Story Number RG. 60-7853. Tape Number: 0086-8400. 
reclutamiento que se hizo con las mujeres y el estado físico y psicológico con el que regresaban.

Llegaron oficiales de la SS y comenzaron a mirar a las mujeres. Las clasificaban por peso, altura y color de cabello; que bubiera variedad para satisfacer todos los gustos. Los nazis engañaron a las mujeres y les decian que si trabajaban seis meses como prostitutas serían liberadas. Además durante ese tiempo recibirian ropa limpia, más comida, cigarrillos y productos de bigiene. Muchas mujeres creyeron esta promesa pero luego se dieron cuenta de que todo era mentira. Después de ser utilizadas las devolvieron desechas a Ravensbrück. Regresaban con el cuerpo roto y los ojos apagados... Perdieron todo atisbo de esperanza. Su mundo fue destruido y no tenian voluntad para vivir ${ }^{51}$.

La dramática situación de los campos y el convencimiento de que todas las prisioneras acabarían tarde o temprano en las cámaras de gas o agotadas por los trabajos forzados explica que algunas mujeres vieran en los Sonderbauten una vía de escape. Romek Dubitzki, superviviente de Auschwitz-Birkenau, justifica la decisión de las jóvenes cuando señala que «si estás en Birkenau y alguien te propone córtate cuatro dedos para ser liberado, lo haces» ${ }^{52}$.

Paralelamente a la selección de las mujeres alemanas, las SS reclutaban a mujeres polacas para nutrir los Sonderbauten de los campos de concentración y exterminio del Este donde los guardianes ucranianos ${ }^{53}$, en virtud de las leyes de pureza racial, no podían mantener relaciones sexuales con mujeres alemanas. Para ellos, las SS establecieron pequeños Sonderbauten en los campos de concentración de Flossenbürg, Buchenwald, el subcampo de Gusen y posiblemente el de Sachsenhausen. En estos campos, y debido que los clientes no eran alemanes, los métodos de explotación de mujeres eran más drásticos y severos. Magdalena Walter, superviviente de Ravensbrück, relata cómo fue reclutada para el Sonderbauten de Buchenwald.

51 Grabación fílmica del Archivo Visual del United States Holocaust Memorial Museum. Story Number RG. 60-7521. Tape Number: 0086-2432.

52 Grabación fílmica del Archivo Visual del United States Holocaust Memorial Museum. Story Number RG. 60-9753. Tape Number: 6521-0943.

53 Los soldados ucranianos eran reclutados entre los prisioneros de guerra soviéticos (POWs) y entrenados en el campo de concentración de Trawniki. Ver GUTMAN, Israel (ed.) (1998): Encyclopedia of the Holocaust. The persecution and murder of the Jews of Europe, Munich, Piper. 
Un día un guardián de las SS me dijo que no fuera a trabajar. Los comandantes de los campos de Ravensbrück y de Buchenwald ordenaron alinear a todas las mujeres. Seleccionaron a las que ellos consideraban más apropiadas para los Sonderbauten y anotaron su número de identificación. Las mujeres escogidas fuimos trasladadas a la enfermería del campo donde tuvimos que desnudarnos ante los guardianes y doctores de las SS. Tras unas semanas de cuarentena, fuimos vestidas con ropa limpia y trasladadas a Buchenwald ${ }^{54}$.

Las declaraciones de las supervivientes permiten conocer las actividades diarias de las mujeres que eran explotadas sexualmente en los Sonderbauten. Las entrevistas realizadas a Magdalena Walter ${ }^{55}$ revelan que el Sonderbauten de Buchenwald era una edificación de madera situada en un área cercana al campo de prisioneros. Estaba estructurado en diversas habitaciones destinadas a acoger a los guardas de las SS, una enfermería, una estancia para las prisioneras reclutadas y una pequeña habitación con camas donde tenían lugar los encuentros sexuales. Las mujeres se despertaban a las 7.30 de la mañana y durante todo el día se ocupaban de los trabajos de limpieza y saneamiento del barracón. A diferencia de lo que ocurría con las demás prisioneras de los campos, ellas estaban exentas de realizar cualquier otro tipo de trabajo y les estaba permitido leer libros no políticos que estaban a disposición del personal de seguridad. Margaret Walter detalla la calma tensa que se vivía en los Sonderbauten durante el día ya que bajo la aparente rutina las jóvenes escondían una ansiedad interior provocada por «las terribles horas que antecedían a los encuentros nocturnos... temblábamos de miedo cuando se acercaba la hora en que los prisioneros terminaban su jornada de trabajo» $^{56}$.

Cada noche teníamos que dejar que los hombres se pusieran encima de nosotras durante dos horas. Esto significa que podian entrar en el barracón, estar con nosotras... Teniamos un baño con varios lavabos... nos lavábamos, entrabamos en la enfermería donde cada noche nos ponian una inyección y después entraba el primer prisionero, y luego el segundo y así... sin parar. Cada prisionero sólo podía estar un cuarto de hora. Cada mujer recibia por

54 Grabación fílmica del Archivo Visual del United States Holocaust Memorial Museum. Story Number RG. 60-7008. Tape Number: 0072-6631.

55 Grabación fílmica del Archivo Visual del United States Holocaust Memorial Museum. Story Number RG. 60-8539. Tape Number: 0072-4318; 7524.

56 Grabación fílmica del Archivo Visual del United States Holocaust Memorial Museum. Story Number RG. 60-9812. Tape Number: 0072-6521 
día entre diezy veinte hombres. Los guardianes de las SS nos vigilaban a través de pequeños agujeros en las puertas ${ }^{57}$.

Algunas supervivientes recuerdan que se sentían incapaces de revelarse ante la situación que vivían. Magdalena Walter relata cómo en una ocasión intentó apuñalar con un cortaúñas al primer hombre con el que debía mantener relaciones sexuales. Sin embargo, en ese momento se dio cuenta de que no había otra elección. Señala que el terror que se vivía en Ravensbrück había destruido su capacidad de resistencia y que todos los prisioneros mostraban una apatía y una muerte en vida que impedía cualquier capacidad de respuesta ${ }^{58}$. En este mismo sentido se pronuncia Linda Bachmann, superviviente del Sonderbauten de Mittelbau-Dora.

Nosotras deciamos: es mejor estar en el Sonderbauten de Ravensbrück que en otro barracón. ¿Qué podiamos hacer? Estábamos en estado de shock... es incomprensible. Estábamos en trance por todo lo que estábamos viviendo. Cuando nos levantábamos por la mañana veíamos que habia un cadáver al lado ... y si se lo comentabas a otro prisionero te decían: «apártalo a un lado». Sólo podias preocuparte de tu propia supervivencia. Nunca pensé que pudiera sobrevivir a aquello... ${ }^{59}$.

El grado de violencia y explotación sexual en los campos de concentración y exterminio anuló las identidades individuales de las mujeres y sesgó cualquier intento de resistencia vital. La mayoría de los testimonios de las supervivientes inciden en la naturalidad con la que se asumía que no existía un futuro fuera de los campos de exterminio. La única meta asumible era la supervivencia diaria; una supervivencia en la que la aceptación de la violencia se convirtió en «una estrategia de vida» en el sentido en el que mientras fueran útiles para el trabajo forzado o para la explotación sexual tendrían asegurada su supervivencia hasta el día siguiente. Esta interiorización de la violencia es el factor que permanece en la conciencia de las supervivientes décadas después de la liberación de los campos. El sentido de culpabilidad, vergüenza y rechazo individual se ve además implementado por el estigma social que recae en ellas por parte de las supervivientes que no participaron en los Sonderbauten. Los muros de los Sonderbauten trazaron una frontera espacial

57 Ibid.

58 Ibid.

59 Grabación fílmica del Archivo Visual del United States Holocaust Memorial Museum. Story Number RG. 60-8900. Tape Number: 0072-1253. 
y simbólica que fomenta la creencia de que las prisioneras recluidas en ellos gozaron de un estatus superior al resto de mujeres. Esta creencia unida a las «relaciones sentimentales» que en ocasiones mantuvieron con prisioneros de alto rango o con cargos intermedios de las SS, fundamentalmente para obtener beneficios como mayores raciones de comida o protección física, despertaron falsas sospechas de colaboracionismo con el régimen nazi que lastró su supervivencia psíquica y emocional tras el fin de la guerra.

\section{A MOdo de CONClusión. LAS hUELLAS DE LA ViolenCia y EXPLOTACiÓN SEXUAL}

La violencia sexual ocasiona unas heridas psíquicas y emocionales en las víctimas del Holocausto que lastran el desarrollo vital de las mujeres incluso décadas después de haber sido liberadas de los campos de concentración y exterminio. Las mujeres que son objeto de explotación sexual en muchas ocasiones desarrollan sentimientos de vergüenza y culpabilidad ${ }^{60}$. Tras el fin de la guerra las mujeres judías encontraron muchas dificultades para rehacer una nueva vida porque el sentimiento de vergüenza por haber sido sometidas a actos de violencia sexual se incrementa con la sensación de culpabilidad que experimentan al recordar que en ocasiones aceptaron la violencia sobre su cuerpo para sobrevivir. Las consecuencias psicológicas de la violencia se agudizan además por el ostracismo social que experimentaron muchas de ellas al ser señaladas públicamente como «prostitutas de las SS», por la infertilidad que les causaron las prácticas sexuales y médicas y por la interiorización de un dolor que muchas mujeres silenciaron durante toda su vida. Es una situación similar a la que acontece con los supervivientes soviéticos que fueron acusados de colaborar con los nazis por el simple hecho de haber sobrevivido a los campos de exterminio. Se crea un recelo social hacia las víctimas que gira en torno a la pregunta de por qué ellos/as han sobrevivido y los demás no. Al margen de las consecuencias psicológicas, algunos informes médicos de las supervivientes de los campos de exterminio establecen una relación entre el encarcelamiento y el desarrollo de enfermedades como estrés crónico, anorexia nerviosa, abortos naturales,

60 VELZEBOER, Marijke (2003): La violencia contra las mijeres. Responde el sector de la salud, Madrid, SPS. 
enfermedades renales, tumores uterinos, pérdida de piezas dentales etc. que dificulta la superación del trauma psicológico ${ }^{61}$.

Sin duda existe un factor historiográfico que ha acentuado el sufrimiento psicológico de las víctimas de explotación sexual. Las primeras investigaciones sobre el Holocausto no investigaron la explotación sexual de las prisioneras en los campos de exterminio. Sus experiencias se invisibilizaron bajo el sufrimiento colectivo que padeció el pueblo judío en su totalidad. El pavor que experimentó el mundo ante el grado de crueldad que alcanzó la política antisemita y racial del NSDAP en el interior de los campos de exterminio fue de tal magnitud que la concreción de la violencia en su vertiente sexual pasó a un segundo plano frente al genocidio masivo y a gran escala. Además, y para facilitar la superación de la guerra, las esferas políticas y sociales designaron consciente o inconscientemente los Juicios de Nüremberg ${ }^{62}$ como un punto de inflexión a partir del cual cimentar la paz. Para ello se intentó olvidar por todas las partes involucradas en el conflicto las experiencias relatadas por los supervivientes de los campos de exterminio obstaculizando así que las mujeres expresasen las vivencias de explotación que habían padecido.

Resulta evidente que la violencia sexual ejercida sobre las mujeres en los campos de concentración y exterminio se enmarca en la política racial nacionalsocialista que intenta obstaculizar la reproducción de las «razas impuras e inferiores» que de alguna manera contaminan biológica y culturalmente la pureza de la «raza aria». La legislación sobre la pureza racial recogida en las Leyes de Núremberg que prohíben las relaciones afectivas y sexuales con personas judías no sólo no protegió a las mujeres judías de los actos de violencia sexual sino que además, bajo las políticas eugenésicas, legitimaron la mutilación de sus cuerpos mediante intervenciones quirúrgicas que perseguían la esterilización forzosa.

61 VELZEBOER, Marijke. Ibid.

62 Proceso jurídico dirigido por el Tribunal Militar Internacional (TMI), coordinado por los países aliados, para depurar y sancionar responsabilidades políticas y militares de los dirigentes, militares y colaboradores del régimen nazi. Se desarrolla entre los años 1945 y 1946 y sentaron jurisprudencia en acusaciones de crímenes contra la humanidad (incluye el genocidio), crímenes de guerra y guerra de agresión. 
El artículo ofrece una visión general de la violencia específica ejercida sobre las mujeres judías en los campos de concentración y exterminio a través de los testimonios ofrecidos por las propias supervivientes. Las mujeres, por el simple de serlo, padecen un tipo de violencia específica que tiene una doble significación política y simbólica y que requiere un análisis que necesariamente debe incorporar una perspectiva de género. El objetivo final del trabajo de investigación es impulsar la perspectiva de género en un tema que historiográficamente no ha ocupado un lugar prominente en las líneas de investigación que conforman la Historia de las Mujeres y los Estudios de Género.

\section{Bibliografía}

BAER, Alejandro (2005): El testimonio audiovisual: imagen y memoria del Holocausto, Madrid, CIS.

BAER, Alejandro (2006): Holocausto. Recuerdo y representación, Madrid, Losada.

BROWNING, Christopher R. (2004): The Origins of the Final Solution: The Evolution of Nari Jewish Policy, September 1939-March 1942. Lincoln: University of Nebraska Press.

BROWNING, Christopher R (2003): Collected Memories: Holocaust History and Postwar Testimony. Madison, Wis, The University of Wisconsin Press, 2003.

BURDS, Jeffrey. «Sexual Violence in Europe in World War II, 1939-1945» in Politics \& Society. 37.1 (2009): 35-73.

COHEN, Beth B (2007): Case Closed: Holocaust Survivors in Postwar America. New Brunswick, N.J. Rutgers University Press. 
DEVILLARD, Marie José \& BAER, Alejandro (2010): «Antropología y Derechos Humanos» [Anthropologie und Menschenrechte] en Revista de Antropología Social, vol 18, pp. 29-51.

DUBA, Ursula (2005): «Not Worth Mentioning» in Journal of Genocide Research, vol. 7 no. 4 (Dec.) (Abingdon). Routledge.

FRIEDMAN, Jonathan C. (2002): Speaking the Unspeakable: Essays on Sexuality, Gender, and Holocaust Survivor Memory. Lanham, M.D. University Press of Americ.

GERLOND, Marion (2005): Off the Road: Remapping Shoab Representations from Perspectives of Ordinary Jewish Women. Thesis (Ph. D.) University of Minnesota.

HERZOG, Esther (2008): Life, death and sacrifice: women and family in the Holocaust, New York, Gefen.

HILBERG, Raul (2003): The Destruction of the European Jews. New Haven, CT: Yale University Press.

HOUSTON, Jaye A (2005): L'Dor V'Dor: Legacies, the Holocaust, Female Survivors, and the Third Generation. Thesis (Ph. D.) Claremont Graduate University.

KERSHAW, Ian (2009): «¿Genocidio improvisado? La aparición de la Solución Final en el Warthegau», en Hitter, los alemanes y la Solución Final, Madrid, La Esfera de los Libros.

KNOBLAUCH, Hubert; BAER, Alejandro; SCHNETTLER, Bernt, et. Al (2008): «Visual Analysis. New Developments in the Interpretative Analysis of Video and Photography» en Forum Qualitative Research (FQS), 9 (3), 2008. 
GUREWITSCH, Brana (1998): Mothers, sisters, resisters. Oral histories of women who survived the Holocaust, London, University of Alabama.

GUTMAN, Israel (ed.) (1998): Encyclopedia of the Holocaust. The persecution and murder of the Jews of Europe, Munich, Piper.

KOONZ, Claudia (2005): La conciencia nazi. La formación del fundamentalismo étnico del III Reich, Barcelona, Paidós.

LEDGEPETH, Sonya \& SAIDEL, Rochelle (2009): Sexual violence against Jewish women during the Holocaust, University Press of New England.

LUNDHOLM, Anja (2004): The gate to hell. Report of a survivor, Rowohlt, Reinbecj debi Hamburg

OFER, Dalia \& WEITZMAN, Leonore (2004): Mujeres en el Holocausto. Fundamentos teóricos para un análisis de género del Holocausto, México, Centro de Investigaciones Interdisciplinarias en Ciencias y Humanidades, UNAM.

ROSEMAN, Mark (2002): La villa, el lago, la reunión. La Conferencia de Wannsee y la Solución Final, Barcelona, RBA.

SCHULZ, Christa (1994): «Female prisioners from Ravesnbrück in brothels of concentrations camps for males», Claus Fullberg (coord.): Women in concentration camps, Bremen, Editions Temmen.

SILVA, Cara de (2008): In memories' kitchen. A legacy from the women in Tereqin, New Jersey, Northvale.

SOMMER, Robert (2010): «Sexual Explotaiton of women in nazi concentration camp brothels», Hedgepeth, S \& Saidel R. (eds): Sexual violence against Jewish women during the Holocaust, Lebanon, Brandeis University Press.

TEDESCHI, Guiliana (2008): There is a place on Earth. A woman in Birkenau, New York, Randhom House. 
VELZEBOER, Marijke (2003): La violencia contra las mujeres. Responde el sector de la salud, Madrid, SPS.

WISTRICH, Robert S. (2002): Hitlery el Holocausto, Barcelona, Mondadori.

\section{Webgrafía}

The United States Holocaust Memorial Museum

http://www.ushmm.org/

USC Shoah Foundation Institute

http://dornsife.usc.edu/vhi/ 